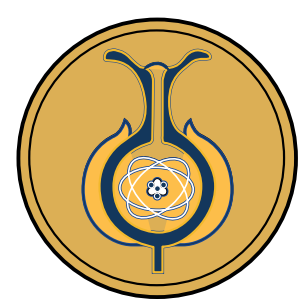

\title{
Análisis de las definiciones de límite que brindan estudiantes universitarios
}

\author{
Analysis of definitions of limit provided by University students
}

Análise das definições de limite fornecidas por estudantes universitários

Yosenith González-Flores ${ }^{1}$, Ana Belén Montoro-Medina ${ }^{2}$, Juan Francisco Ruiz-Hidalgo ${ }^{2}$

Received: Dec/1/2020 • Accepted: Apr/9/2021 • Published: Jul/31/2021

\section{Resumen}

Este trabajo tiene un doble objetivo; por un lado, analizar las definiciones que brindan estudiantes universitarios sobre el concepto de límite en un punto de una función y, por otro, tanto diseñar como validar un sistema de categorías para llevarlo a cabo. Se estudiaron las definiciones de límite proporcionadas por 38 estudiantes universitarios de Biología e Ingeniería en Química Industrial, matriculados en un curso de Cálculo Diferencial e Integral, durante el primer semestre del 2018, en la Universidad Nacional, Costa Rica. Para ello, se utilizaron categorías a priori y fue necesario complementarlas con otras de naturaleza inductiva, surgidas durante el análisis de contenido de las respuestas. Estas categorías fueron ratificadas mediante un análisis de fiabilidad. Los estudiantes evidenciaron la categoría límite como objeto en la mitad de sus respuestas, aproximadamente, y la categoría límite como proceso en más de tres cuartas partes. Además, constataron las categorías términos de posición relativa y descoordinación general de los procesos, en un estimado de la mitad de sus respuestas. El sistema de categorías creado permitió examinar unidades de información de una manera ordenada, simple y replicable. Finalmente, los estudiantes muestran una concepción dual del límite ya sea como un objeto, noción fija y estática, o como un proceso, noción procesual y dinámica. Asimismo, consideramos que el análisis de contenido descrito en la metodología puede permitir a futuros investigadores crear un sistema de categorías similar o usar este para indagaciones en otros contextos.

Palabras clave: Concepto de límite; cálculo; contenido matemático, didáctica del análisis matemático; estudiantes universitarios; pensamiento matemático avanzado.

\section{Abstract}

This paper serves two objectives: analyze the definitions provided by college students for the concept of limit at a point in a function as well as design and validate a category system to carry it out. The definitions of limit were provided by 38 university students in the Biology and Engineering in Industrial Chemistry program, enrolled in a Differential and Integral Calculus course during the first semester of 2018, at Universidad Nacional, Costa Rica. A priori categories were used and complemented with other categories of an inductive nature, which emerged during the analysis of the responses. These categories were validated

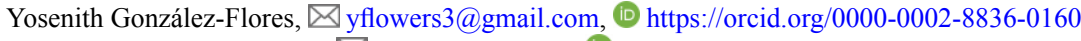

Ana Belén Montoro-Medina, $\$ amontoro@ugr.es, (D) https://orcid.org/0000-0001-9344-5778

Juan Francisco Ruiz-Hidalgo, \jfruiz@ugr.es, (1) https://orcid.org/0000-0002-4805-6922

1 Escuela de Matemática, Universidad Nacional, Heredia, Costa Rica.

2 Departamento de Didáctica de la Matemática, Universidad de Granada, Granada, España. 
through a reliability analysis. Approximately half of the students' answers showed the limit category as an object and over three-quarters showed the limit category as a process. In addition, approximately half of their answers showed the categories terms of relative position and general lack of coordination of the processes. The category system created allowed for the analysis of information units in an organized, simple, and replicable way. Finally, students showed a dual conception of limit either as an object, fixed and static notion, or as a process, processual and dynamic notion. Furthermore, the content analysis described in the methodology may allow future researchers to create a similar category system or use this one for research in other contexts.

Keywords: limit concept; calculus; mathematical content; didactics of mathematical analysis; university students; advanced mathematical thinking

\section{Resumo}

Este trabalho tem um objetivo duplo: por um lado, analisar as definições que estudantes universitários oferecem sobre o conceito de limite em um ponto de uma função e, por outro lado, desenhar e validar um sistema de categorias para realizá-lo. Foram estudadas as definições de limite proporcionadas por 38 estudantes universitários de Biologia e Engenharia em Química Industrial, matriculados em uma cadeira de Cálculo Diferencial e Integral, durante o primeiro semestre de 2018, na Universidade Nacional, Costa Rica. Para isso, foram utilizadas categorias a priori e foi preciso complementá-las com outras de natureza indutiva, surgidas durante a análise de conteúdo das respostas. Estas categorias foram ratificadas por médio de uma análise de fiabilidade. Os estudantes demonstraram a categoria limite como objeto na metade de suas respostas, a proximadamente, e a categoria limite como processo em mais de três quartos. Também, constataram as categorias termos de posição relativa e descoordenação geral dos processos, em uma média da metade de suas respostas. 0 sistema de categorias criado permitiu examinar unidades de informação de uma maneira estruturada, simples e replicável. Finalmente, os estudantes mostram uma concepção dual do limite, seja como um objeto, noção fixa e estática, ou como um processo, noção processual e dinâmica. Igualmente, consideramos que a análise de conteúdo descrito na metodologia pode permitir que futuros pesquisadores criem um sistema de categorias semelhante ou usem este para indagações em outros contextos.

Palavras-chave: conceito de limite; cálculo; conteúdo matemático; didática da análise matemática; estudantes universitários; pensamento matemático avançado.

\section{Introducción}

Esta investigación forma parte de la didáctica del análisis matemático, particularmente del pensamiento matemático avanzado, ámbito en el que destacan procesos cognitivos abstractos como definir, demostrar y formalizar (Azcárate y Camacho, 2003). El concepto matemático de límite tiene una posición central que impregna el análisis matemático, como fundamento de la teoría de la aproximación, de la continuidad y del cálculo diferencial e integral (Cornu, 2002; Kidron, 2014). Se trata de una noción cuya comprensión es compleja, en cuyo aprendizaje por estudiantes se observa una reincidencia de conflictos cognitivos o dificultades (Artigue, 1995; Blázquez y Ortega, 1998; Blázquez y Ortega, 2002; Juter, 2007a; Juter, 2007b; Romero, 1997; 
Sierpinska, 1987; Tall, 1980; Tall y Katz, 2014; Tall y Vinner, 1981; Vrancken, Gregorini, Engler, Muller, y Hecklein, 2006).

Una de las principales dificultades en su enseñanza y aprendizaje radica, no solo en su riqueza y complejidad, sino también en la medida en que los aspectos cognitivos no pueden generarse únicamente a partir de la definición matemática. La distinción entre la definición y el concepto en sí es didácticamente muy importante (Cornu, 2002). Esta complejidad ha generado que, desde la didáctica de la matemática, surjan investigaciones que buscan indagar en distintos aspectos de la comprensión del límite, entre ellas destacan algunos estudios como los de Kidron y Tall (2014), Swinyard (2011) y Tall y Katz (2014).

Algunas investigaciones sobre las concepciones del límite en estudiantes de secundaria y de universidad evidencian que muchos tienen una noción intuitiva del límite, que describen con términos como tender, aproximar, alcanzar, rebasar y límite. En algunas ocasiones, estos términos son empleados en clase por los docentes, y los estudiantes los usan con un sentido cotidiano que no necesariamente coincide con el significado matemático (Blázquez,1999; Blázquez, 2000; Fernández-Plaza, Ruiz-Hidalgo, Rico, y Castro, 2013; Monaghan, 1991; Williams, 1991). En algunos casos, estas concepciones y los términos empleados por los estudiantes pueden relacionarse con concepciones de carácter epistemológico planteadas por matemáticos tales como D'Alembert, quien afirmaba que el límite no se puede alcanzar y Cauchy para quien es alcanzable (Sierra, González, y López, 2000).

Fernández-Plaza et al. (2013) describieron e interpretaron las definiciones de estudiantes de bachillerato sobre el concepto de límite finito de una función en un punto en términos de aspectos estructurales, compilados y sintetizados de investigaciones previas. Para ello, plantearon nueve categorías: tipo de objeto/proceso, vinculación entre límite e imagen, descoordinación de los procesos en el dominio y en el rango de la función, referencia explícita a un sistema de representación distinto al numérico o simbólico, evaluación en el punto (sustitución directa), tabla de valores, condiciones de lateralidad y doble convergencia, aspectos estructurales de alcanzabilidad y rebasabilidad, reproducción de la definición de referencia. Este trabajo fue nuestro punto de partida, ya que tomamos algunas de sus categorías tal cual, otras las adaptamos y elaboramos algunas nuevas.

En Costa Rica, el primer contacto de los estudiantes con el concepto de límite se realiza en la Universidad. Particularmente, en la Universidad Nacional (UNA), la primera asignatura en la que se inserta es Cálculo I, asignatura en la que se estudian límites, derivadas, aplicaciones de la derivada, integrales y aplicaciones de la integral y que se oferta en las carreras de ingenierías, ciencias experimentales, economía, administración y enseñanza de las ciencias. Este curso no tiene un enfoque formal, por tanto, la presentación de los conceptos se hace de forma intuitiva, sin hacer uso de definiciones formales ni demostraciones matemáticas de los resultados.

Este estudio forma parte de uno más amplio cuya finalidad es analizar el significado que le atribuyen estudiantes universitarios de la UNA al concepto de límite en un punto de una función en una variable, en el que queremos explorar el significado que tienen los estudiantes durante la enseñanza del tema en el curso de Cálculo I y cómo se modifica al terminar dicha enseñanza. El significado de un contenido matemático es 
ampliamente estudiado; de todos los marcos existentes, consideramos el posicionamiento teórico de Rico y colaboradores (Rico, 2012, 2013, 2016a, 2016b) que lo consideran desde una postura filosófica y curricular. Dentro de este marco, nos centramos únicamente en este artículo en uno de los elementos que forman parte del significado: las definiciones. En concreto, se analizan las definiciones del concepto de límite proporcionadas por 38 estudiantes de Biología e Ingeniería en Química Industrial durante su primer contacto con dicho concepto.

Las dificultades en la aplicación de las categorías del estudio de Fernández-Plaza et al. (2013) a todas las respuestas de nuestro trabajo nos llevaron a diseñar y validar un sistema de categorías, adaptadas de dicho estudio. Los resultados muestran que se trata de un sistema de categorías fiable que permite analizar definiciones del concepto de límite de manera ordenada, sistemática y replicable. Resaltamos la concepción dual objeto/proceso que atribuyeron los estudiantes al límite, pues aproximadamente en la mitad de las respuestas lo señalaron como objeto y en más de tres cuartas partes de las respuestas lo señalaron como proceso. Además, en más de la mitad de las respuestas evidenciaron las categorías términos de posición relativa y descoordinación general de los procesos. Para analizar el significado del concepto de límite, específicamente las definiciones, requerimos un marco teórico que ahonde en el significado de un contenido matemático escolar. A continuación, en el siguiente apartado, hacemos una descripción del marco que hemos utilizado.

\section{Marco teórico}

La noción fundamental que soporta el marco teórico es la de significado de un contenido matemático escolar. Esta noción ha sido ampliamente estudiada y desarrollada en matemática educativa por autores como Steinbring (1997, 2006), Vergnaud (2009, 2013), Kilpatrick, Hoyles y Skovsmose (2005), Thompson (2013, 2016), Thompson y Milner (2019), o Byerley y Thompson (2017). En este estudio se asume la noción de significado establecida por Rico (Rico, 2012, 2013, 2016a, 2016b), que tiene un doble fundamento filosófico y curricular. Por un lado, se basa en la consideración de significado introducida por Frege a finales del siglo XIX, que establece la diferencia entre signo y significado, $y$ a su vez en el significado discrimina entre referencia y sentido. Así, introduce la noción de triángulo semántico a través del signo o término con el que se expresa, gráficos y notaciones que lo representan; por su referencia o concepto propiamente y la estructura lógica en la que se inserta; y por su sentido o modo en que vienen dados los objetos que encajan en el concepto, o modos de uso con que puede ser entendido, aplicado e interpretado. Este trabajo ha sido transformado y desarrollado para estudiar los significados de conceptos y contenidos de las matemáticas escolares (Rico, 2012, 2013, 2016a, 2016b; Rico y Ruiz-Hidalgo, 2018); considera, para su análisis, tres componentes: estructura conceptual, sistemas de representación, y sentidos y modos de uso (Figura 1).

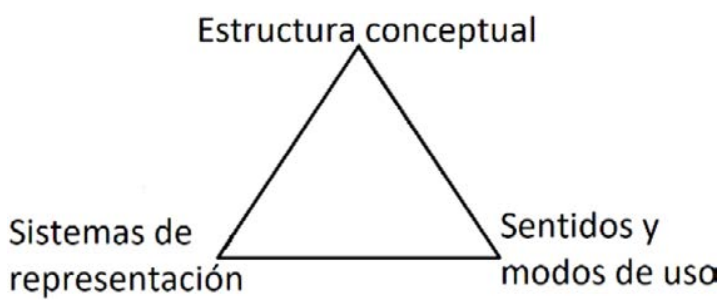

Figura 1. Triángulo semántico de un concepto matemático escolar. Adaptado de Rico (2012). 
Por otro lado, desde una óptica más curricular y cognitiva, la noción de significado que hemos considerado se basa en la dualidad del conocimiento matemático establecida por Bell, Costello \& Küchemann (1983), quienes indican que, al discutir la comprensión y la enseñanza de las matemáticas, debemos distinguir entre conceptos y estrategias. Posteriormente, Hiebert y Lefevre (1986) señalan que la distinción entre conceptos y procedimientos juega un papel importante en la adquisición de conocimientos. El conocimiento conceptual se caracteriza por ser un conocimiento rico en relaciones, puede considerarse como una red de conocimiento en la que las relaciones de enlace son tan prominentes como las piezas discretas de información. El desarrollo del conocimiento conceptual se logra mediante la construcción de relaciones entre piezas de información. En cambio, el conocimiento procedimental está compuesto, por un lado, por el lenguaje formal, o sistema de representación de símbolos de las matemáticas $\mathrm{y}$, por otro, de algoritmos o reglas para completar tareas matemáticas. Sobre este doble fundamento, consideramos que conocer el significado de un concepto matemático implica saber "su definición, representarlo, mostrar sus operaciones, relaciones y propiedades y sus modos de uso, interpretación y aplicación a la resolución de problemas" (Rico, 2016a, p. 94). A continuación, se detallan las tres componentes del significado de un contenido matemático escolar, en cuanto a los aspectos estructurales que resultan centrales de este trabajo.

\section{Estructura conceptual}

Comprende los conceptos, procedimientos, propiedades, argumentos y proposiciones que se obtienen y sus criterios de veracidad, vinculados con un contenido matemático, junto con la estructura formal que proporciona referencia a los contenidos utilizados (Rico, 2012). La estructura conceptual se organiza en tres componentes: campo conceptual, campo procedimental y campo actitudinal.

El campo conceptual es el conglomerado de conceptos y relaciones que hacen referencia a un contenido matemático. Se reconocen tres niveles de conocimiento: los hechos, que se componen de términos, notaciones, convenios y resultados; los conceptos, que describen una regularidad de un conjunto de hechos; y las estructuras conceptuales, que corresponden a un conjunto de conceptos y transformaciones relacionados entre sí. (Fernández-Plaza, 2016; Hiebert y Lefevre, 1986; Rico, 1997).

El campo procedimental comprende las reglas, operaciones, algoritmos, propiedades, sus formas de procesamiento y el conocimiento implicado al resolver una tarea matemática. Según la complejidad del contenido matemático, encontramos tres niveles: las destrezas, que son el procesamiento de hechos; los razonamientos, que son el procesamiento de conceptos; y las estrategias, que implican el procesamiento de una o varias estructuras conceptuales (Fernández-Plaza, 2016; Hiebert y Lefevre, 1986; Rico, 1997).

El campo actitudinal se describe en tres categorías en las matemáticas escolares: las emociones, que comprenden la seguridad, la disciplina, el dominio y autoestima; la moralidad y las normas, que implican el respeto y la aplicación de reglas, la corrección de procedimientos y la coherencia; y los valores éticos (Bell, Costello \& Küchemann, 1983, Fernández-Plaza, 2016; Rico, 1997). 


\section{Sistemas de representación}

Las representaciones aluden a signos, notaciones simbólicas o gráficas para cada noción matemática que expresan los conceptos y procedimientos, así como sus relaciones, características y propiedades. Se pueden considerar dos grupos de sistemas de representación: las simbólicas que comprenden símbolos alfanuméricos, y las gráficas que aluden a figuras (Castro y Castro, 1997; Kaput, 1987). Se pueden considerar representaciones verbales, se trata del uso del lenguaje escrito para referirse a conceptos, procedimientos y propiedades; gráficas, que alude al uso de figuras en el plano cartesiano; simbólica, que resalta el uso de números, símbolos matemáticos y del abecedario en una organización no tabular; y tabular, que describe al uso de símbolos alfanuméricos organizados en una tabla.

\section{Sentidos y modos de uso}

Los sentidos y modos de uso de los conceptos matemáticos hacen referencia a las diversas situaciones a las que responde, a los problemas que resuelve y a los fenómenos que organizan lo que permite complementar sus significados (Ruiz-Hidalgo, 2016). Se consideran relevantes tres elementos: los términos y modos de uso, que son palabras que sintetizan la definición de un concepto matemático para brindarle sentido, corresponden a distintas interpretaciones de este; los contextos matemáticos, que están relacionados con las funciones y las cuestiones a las que responden los conceptos matemáticos; y las situaciones que corresponden a las circunstancias o condiciones en las que se aplica y trabaja el concepto matemático.

\section{Objetivos del estudio}

Basados en el marco teórico y en los antecedentes, concretamos dos objetivos para el trabajo: (1) realizar el diseño y validación de un sistema de categorías para el análisis de definiciones de límite de una función en un punto, basadas en el trabajo de Fernández-Plaza et al. (2013); y (2) utilizar el sistema de categorías para analizar las definiciones del concepto de límite proporcionadas por 38 estudiantes universitarios de Biología e Ingeniería en Química Industrial de la UNA. Para realizar el análisis de las definiciones del concepto de límite decidimos utilizar el análisis de contenido como método de investigación, que se muestra con detalle en el siguiente apartado.

\section{Metodología}

El estudio es cualitativo, con alcance exploratorio y descriptivo (Hernández, Fernández y Baptista, 2014), ya que no se conocen antecedentes de investigaciones similares en Costa Rica y se pretende analizar las definiciones que brindan los estudiantes sobre el concepto de límite sin emitir juicios de valor. Para analizar la información, la herramienta metodológica que vamos a utilizar es el análisis de contenido, que posibilita codificar y categorizar las unidades de análisis en instrumentos de respuesta abierta, comparar las categorías y establecer vínculos entre ellas y extraer conclusiones teóricas (Cohen, Manion y Morrison, 2007). Es una técnica de investigación para hacer inferencias replicables y válidas de textos a los contextos de su uso. Implica procedimientos especializados, proporciona nuevas ideas, aumenta la comprensión del investigador de fenómenos particulares o informa acciones prácticas. Es una herramienta 
científica (Krippendorff, 2004). No hay un método aceptado universalmente para realizar el análisis de contenido, por lo que seguimos a Rico y Fernández-Cano (2013) que manifiestan que, en general, el análisis de contenido tiene siete fases que se describen en la Figura 2.

Fases 1, 2 y 3: En nuestro caso, el contenido a analizar en este trabajo corresponde a la producción escrita que brindaron los 38 estudiantes de Cálculo I de Biología e Ingeniería en Química Industrial durante el primer semestre del 2018 al pedirles que explicaran, con sus propias palabras, el significado de la definición de límite que les había dado su profesor. Con ello buscábamos conocer, en aspectos generales, la forma en que expresaban su interpretación de dicho concepto y los aspectos o características que resaltaban de este mismo. En este sentido, consideramos cada definición dada por los estudiantes como una unidad de análisis, entendiendo que una definición completa acaba cuando el estudiante agrega un punto y aparte. Once estudiantes respondieron dos veces a la tarea, por lo que en total trabajamos con 49 unidades de análisis.

Fase 4. Para analizar las respuestas de los estudiantes partimos de las categorías de análisis propuestas por Fernández-Plaza et al. (2013). Más adelante, al poner en práctica este sistema de categorías con las respuestas de los estudiantes (fase 5), surgió la necesidad de precisar algunas de ellas e incluir categorías nuevas. En la Tabla 1 presentamos la relación entre estas categorías y las categorías que emergieron de nuestro estudio. Hay que destacar que todas estas categorías son dicotómicas e indican la presencia o ausencia de la característica que describe la categoría. Dado que el diseño y validación de este sistema de categorías supone uno de nuestros objetivos de investigación, la descripción definitiva de cada una de nuestras categorías se detallará en el apartado descripción de las categorías de análisis. Posteriormente, en el apartado fiabilidad del análisis de las categorías, se describirá el proceso de validación.

Fase 1: Delimitar el contenido (texto, discurso o producción escrita) a analizar.

Fase 2: Precisar la unidad de análisis (palabra, frase o párrafo).

Fase 3: Ubicar o inferir en el texto las unidades de análisis.

Fase 4: Definir e interpretar las categorías consideradas.

Fase 5: Codificar y cuantificar a través de frecuencias o rangos las unidades de análisis adscritas previamente al sistema de categorías establecido.

Fase 6: Vincular e interpretar las categorías considerando sus unidades de análisis adscritas.

Fase 7: Relacionar el proceso de análisis de contenido con lo que se indaga y con los agentes que intervienen: hablante - escritor $\mathrm{u}$ oyente - lector.

Figura 2. Fases del análisis de contenido.

Adaptado de Rico y Fernández-Cano (2013). 
Tabla 1. Adaptación de las categorías de Fernández-Plaza et al. (2013)

\begin{tabular}{|c|c|}
\hline Categorías de Fernández-Plaza et al. (2013) & Categorías de nuestro estudio \\
\hline \multirow[t]{2}{*}{ Tipo de objeto/proceso } & Límite como objeto (LO) \\
\hline & Límite como proceso (LP) \\
\hline $\begin{array}{l}\text { Vinculación entre límite e imagen (identificación, } \\
\text { conexión e independencia) }\end{array}$ & Vinculación entre límite e imagen (LI) \\
\hline \multirow[t]{4}{*}{$\begin{array}{l}\text { Descoordinación de los procesos en el dominio y en } \\
\text { el rango de la función }\end{array}$} & $\begin{array}{l}\text { Descoordinación de los procesos en el dominio de la } \\
\text { función (DX) }\end{array}$ \\
\hline & $\begin{array}{l}\text { Descoordinación de los procesos en el rango de la } \\
\text { función (DY) }\end{array}$ \\
\hline & Descoordinación general de los procesos (DG) \\
\hline & Coordinación entre las variables $x$ e $y(\mathrm{CV})$ \\
\hline \multirow{2}{*}{$\begin{array}{l}\text { Referencia explícita a un sistema de representación } \\
\text { distinto al numérico o simbólico }\end{array}$} & Símbolos matemáticos y notaciones (S) \\
\hline & $\begin{array}{l}\text { Referencia a un sistema de representación gráfico o } \\
\text { geométrico }(\mathrm{G})\end{array}$ \\
\hline Evaluación en el punto & ---- \\
\hline Tabla de valores & ---- \\
\hline Condiciones de lateralidad y doble convergencia & $\begin{array}{l}\text { Condiciones de lateralidad y doble convergencia } \\
\text { (CLDC) }\end{array}$ \\
\hline \multirow[t]{2}{*}{ Aspectos estructurales de alcanzabilidad y rebasabilidad } & Aspectos estructurales de no alcanzabilidad (NA) \\
\hline & $\begin{array}{l}\text { Aspectos estructurales de alcanzabilidad y no rebasab- } \\
\text { ilidad (ANR) }\end{array}$ \\
\hline \multirow[t]{4}{*}{ Reproducción de la definición de referencia } & ---- \\
\hline & Referencia a las variables $x$ e $y(\mathrm{RV})$ \\
\hline & Términos de posición relativa (PR) \\
\hline & Propiedades matemáticas (PM) \\
\hline
\end{tabular}

Nota: Fuente propia de la investigación.

En la Tabla 1 se muestra la correspondencia entre las categorías de este estudio y las de Fernández-Plaza et al. (2013). La categoría tipo de objeto/proceso es "cuando los estudiantes establecen distintas referencias para el objeto límite (lugar del plano, conjunto de puntos, recta, etc.); en algunos casos, se destaca también su dualidad procesual (aproximación)" (p. 122), se convirtió en dos categorías que no son excluyentes: límite como objeto y límite como proceso. La categoría vinculación entre límite e imagen se establece cuando el estudiante "asigna al límite un valor de imagen se observa una Identificación (de manera general), una Conexión (en casos particulares) o bien una Independencia entre dicho límite y el valor imagen de la función" (p. 122), se convirtió en una única categoría llamada vinculación entre límite e imagen, porque lo que nos interesa es determinar si los estudiantes relacionan el límite con la imagen, ya sea de manera general o con casos particulares.

La categoría descoordinación de los procesos en el dominio y en el rango de la función, que se presenta cuando "los escolares sólo se refieren a la variable $x$ ” (p. 122), motivó la creación de cuatro categorías mutuamente excluyentes: coordinación entre las variables $x$ e $y$, descoordinación de los procesos en el dominio de la función, descoordinación de los procesos en el rango de la función y descoordinación general de los procesos. 
Asimismo, la categoría denominada referencia explícita a un sistema de representación distinto al numérico o simbólico se presenta con el "empleo de términos para el objeto/proceso límite relacionados con un sistema de representación diferente al numérico o simbólico" (p. 122). Esta motivó la creación de la categoría símbolos matemáticos y notaciones, y de la categoría referencia a un sistema de representación gráfico o geométrico.

La categoría condiciones de lateralidad y doble convergencia, en la que los estudiantes "expresan que los procesos de cálculo del límite, bien por la izquierda o bien por la derecha, deben dar el mismo resultado" (p. 122) se mantiene sin modificaciones. La categoría aspectos estructurales de alcanzabilidad y rebasabilidad, descrita como "la posibilidad o no de alcanzar o rebasar el límite puede ser expresada por los escolares en sus definiciones” (p. 122), se desglosó en dos categorías: no alcanzable y alcanzable pero no rebasable. Finalmente, surgió la necesidad de incluir las categorías referencia a las variables $x$ e $y$, términos de posición relativa y propiedades matemáticas.

Fase 5. Una vez establecidas las categorías, se procedió al análisis de la presencia o ausencia de cada una de las características descritas en la categoría. Los datos se anotaron en una hoja de cálculo en Excel que contiene una columna para cada una de las 15 categorías, correspondiendo cada fila a una unidad de información. Se agregaba 1 cuando estaba presente la categoría en la unidad de información y 0 cuando no lo estaba.
La Tabla 2 muestra el resultado de la codificación de la unidad de análisis EBM0125 "El límite de $f$ en $a$ es $L$, solo cuando si al aproximarse a $a$ por derecha y por izquierda se obtiene el resultado de que las imágenes de $a$ en $y$ tienden a $L$ ", en la que se encuentra la dualidad del límite como objeto, ya que indica que "el límite de $f$ en $a$ es $L$ ", y como proceso, cuando utiliza los términos "aproximarse" y "tienden". Además, identifica el límite con la imagen, hay una coordinación entre las variables $x \mathrm{e}$ $y$, establece la necesidad de que las aproximaciones por la derecha y la izquierda del número coincidan (CLDC), utiliza términos de posición relativa, referencia a las variables (imágenes) y usa lenguaje simbólico $(f$, $a, L, y)$. Esta codificación fue realizada de manera independiente por los tres investigadores. Posteriormente, se calcularon las frecuencias absolutas y porcentajes de cada una de las categorías. Los resultados de dicho análisis se presentarán en el apartado análisis y resultados.

Fases 6 y 7, relativas a la vinculación e interpretación de las categorías y a la relación del análisis realizado con lo que se indaga y los agentes que intervienen, se expondrán en el apartado de conclusiones.

\section{Descripción de las categorías de análisis}

Presentamos la definición de cada una de las categorías de nuestro estudio expuestas en la segunda columna de la Tabla 1.

(1) Límite como objeto (LO): los sujetos establecen distintas referencias para el

Tabla 2. Categorización de una unidad de información

\begin{tabular}{ccccccccccccccc}
\hline LO & LP & LI & CV & DX & DY & DG & CLDC & PR & NA & ANR & RV & S & G & PM \\
\hline 1 & 1 & 1 & 1 & 0 & 0 & 0 & 1 & 0 & 0 & 1 & 1 & 1 & 0 & 0 \\
\hline
\end{tabular}

Nota: Fuente propia de la investigación. 
objeto límite (Fernández-Plaza et al., 2013). Se entiende límite como una noción estática. Algunas palabras claves para identificar esta categoría son: número, valor, lugar, etc. Si un sujeto dice que un límite se aproxima a un número, pero no dice que el límite sea el número, entonces no hay evidencia de LO.

(2) Límite como proceso (LP): los sujetos refieren al límite de manera procesual (aproximación) (Fernández-Plaza et al., 2013). Es una noción dinámica. Los sujetos hacen referencia a un proceso de obtención o procedimiento. Algunas palabras claves para identificar esta categoría son: aproximarse, tiende, acercar, etc. Si el sujeto usa un verbo de procedimiento, y no dice nada sobre el objeto límite, asumimos que evidencia LP, como por ejemplo "estudia lo que ocurre alrededor de los puntos", "análisis de un punto". Las categorías límite como objeto y límite como proceso no son mutuamente excluyentes.

(3) Vinculación entre límite e imagen (LI): los sujetos atribuyen al límite un valor de imagen de la función (Fernández-Plaza et al., 2013). La alusión a la imagen puede hacerse de manera implícita, por ejemplo, en la afirmación “es el valor máximo o más próximo al punto que pertenece a una función" dice que pertenece a una función, por ende, ese valor debe ser imagen, "el número al que tiende una función cuando sustituyo valores en una variable específica", pues al sustituir los valores se entiende que son las imágenes. Cuando los sujetos vinculan el límite con un valor de la imagen en particular, están evidenciando el límite como objeto, es decir, si se da LI entonces se da LO.

Las categorías 4-7 representan las distintas posibilidades que encontramos en lo relativo a la coordinación o descoordinación entre las variables $x$ e $y$, por lo que son mutuamente excluyentes. Además, estas categorías solo se evidencian cuando los sujetos aluden al límite como proceso.
(4) Coordinación entre las variables $x$ e $y(\mathrm{CV})$ : los sujetos indican que es un proceso en el que están implicadas las dos variables $(x$ e $y$ ), una depende de otra. Los sujetos evidencian convergencia de $y$ en relación con la convergencia de $x$, es la aproximación de las imágenes a un número, cuando $x$ se aproxima al punto.

(5) Descoordinación de los procesos en el dominio de la función (DX): los sujetos, de manera explícita, solo evidencian convergencia de $x$, es decir, únicamente evidencian la aproximación de las preimágenes a un número. (Fernández-Plaza et al., 2013).

(6) Descoordinación de los procesos en el rango de la función (DY): Los sujetos, de manera explícita, solo evidencian convergencia de $y$, es decir, solo evidencian la aproximación de las imágenes a un número.

(7) Descoordinación general de los procesos (DG): Los sujetos evidencian convergencia en una sola de las variables, ya sea en la variable independiente o en la variable dependiente, pero no explicitan en cuál de ellas. Es decir, hay descoordinación general, cuando el estudiante ve al límite como un proceso, no coordina las variables, no descoordina en $x$, no descoordina en $y$.

(8) Condiciones de lateralidad y doble convergencia (CLDC): los sujetos expresan que los procesos de cálculo del límite, por la izquierda y por la derecha, deben dar el mismo resultado (Fernández-Plaza et al., 2013).

(9) Términos de posición relativa (PR): los sujetos usan palabras o frases para el límite que denotan una posición relativa, es decir, aluden a dónde está ubicada una noción matemática con respecto a una noción fija (objeto) de manera explícita o implícita. Algunas palabras claves para identificar esta categoría son: cercano, a la derecha, encima de, mayor que, próximo, por encima, es una proximidad. Esta categoría no 
necesariamente está relacionada con LO. Además, si los sujetos evidencian la categoría CLDC entonces señalan la categoría PR, $\mathrm{y}$ puede haber PR sin que haya CLDC.

Las categorías 10-11 son mutuamente excluyentes.

(10) Aspectos estructurales de no alcanzabilidad (NA): los sujetos expresan la imposibilidad de alcanzar el límite. (Fernández-Plaza et al., 2013). Algunas frases claves para identificar esta categoría son: sin nunca tocar dicho valor, siguiendo la regla de que, $y$ no va a ser el resultado, nunca lo llega a pasar o tocar, etc.

(11) Aspectos estructurales de alcanzabilidad y no rebasabilidad (ANR): los sujetos expresan la posibilidad de alcanzar el límite, pero no de rebasarlo o sobrepasarlo. Usan palabras como no pasar (Fernández-Plaza et al., 2013). Algunas frases claves para identificar esta categoría son: punto final, valor máximo, hasta donde llega la función.

(12) Referencia a las variables $x$ e $y$ (RV): los sujetos usan palabras específicas para aludir a las variables independiente y dependiente de la función. Algunas palabras claves para identificar esta categoría son: preimagen, imagen, punto en $x$, punto en $y$, etc.

(13) Símbolos matemáticos y notaciones (S): los sujetos usan símbolos matemáticos, notaciones para aludir a diferentes nociones matemáticas. Algunos símbolos claves para identificar esta categoría son: $f$, $a, L, x, y$, etc.

(14) Referencia a un sistema de representación gráfico geométrico $(\mathrm{G})$, es decir, los sujetos usan términos para el objeto/proceso límite relacionados con un sistema de representación gráfico o geométrico. Algunas palabras claves para identificar esta categoría son: punto final, en la gráfica, el punto en una recta, huecos que hay en un gráfico, etc.
(15) Propiedades matemáticas (PM): los sujetos hacen referencia a propiedades, o nociones que son verdaderas en la matemática no contempladas en el resto de las categorías de análisis.

\section{Fiabilidad del análisis de las categorías}

La fiabilidad es la medida en que un procedimiento de medición arroja los mismos resultados en ensayos repetidos. Esto último es particularmente relevante para el análisis de contenido codificado por seres humanos, ya que una medida es poco valiosa si solo puede ser realizada una vez o por una sola persona en particular (Neuendorf, 2017). La replicabilidad es la forma más importante de fiabilidad (Krippendorff, 2004). Los índices de fiabilidad se utilizan para determinar la fiabilidad de las mediciones realizadas por los investigadores (Sim \& Wright, 2005).

Neuendorf (2017) describe cuatro amenazas para la fiabilidad: un esquema de codificación mal ejecutado, el entrenamiento inadecuado del codificador, la fatiga del codificador y deriva del codificador y, la presencia de un codificador deshonesto. Para evitar estas amenazas, hemos realizado varios ciclos de revisión de las categorías con una muestra de las respuestas de los estudiantes y, posteriormente, hemos discutido los resultados, como parte del entrenamiento en la codificación. Este proceso ha dado lugar a la modificación de la descripción de las categorías, que hemos redactado de la forma más explicativa posible, incluyendo términos usuales en los que aparecen y algunas aclaraciones a dudas que surgieron al aplicar dichas categorías a una muestra. Además, hemos asociado las que están relacionadas para que sea más sencilla la categorización. Por último, dada la importancia que tiene para nosotros ajustarnos a la realidad, hemos realizado las categorizaciones 
en momentos que nos sintamos cómodos y centrados, evitando dedicarnos a este trabajo cuando estábamos fatigados.

Como se indicó en la metodología, tras analizar de manera independiente 10 respuestas de los estudiantes utilizando las categorías de Fernández-Plaza et al. (2013), nos dimos cuenta de que esas categorías no eran suficientes para analizar las respuestas, por lo que establecimos las categorías descritas en el apartado anterior. Con estas 15 categorías analizamos las respuestas de los estudiantes del curso de Biología de manera independiente y realizamos una triangulación para consensuar, ajustar y explicar más las definiciones de las categorías de manera que nos permitieran hacer un análisis sistemático y con la menor de las ambigüedades. A modo de ejemplo, en la categoría LI se agregó que la alusión a la imagen se podía hacer de manera implícita, por ejemplo, en la afirmación "es el valor máximo o más próximo al punto que pertenece a una función" dice que pertenece a una función, por ende, ese valor debe ser imagen, "el número al que tiende una función cuando sustituyo valores en una variable específica", pues al sustituir los valores se entiende que son las imágenes, ya que uno de los investigadores solo anotaba esta categoría cuando aludía a la imagen explícitamente. Algo similar consensuamos para la categoría LO, ya que acordamos que, si un estudiante dice que un límite se aproxima a un número, pero no dice que el límite sea el número, entonces no está presente dicha categoría. Por limitaciones de espacio, no podemos desglosar los cambios de todas las categorías. Una vez depuradas las 15 categorías, los tres investigadores analizamos nuevamente, de manera independiente, las 49 respuestas de los
38 estudiantes y determinamos nuestro grado de acuerdo en las categorizaciones de las respuestas de los sujetos de investigación.

Para esto hemos utilizado el acuerdo de categorización entre evaluadores, debido a que medimos el acuerdo entre los tres investigadores. En concreto, utilizamos el índice Holsti, que se entiende como el porcentaje de acuerdo entre codificadores de los mismos casos (Neuendorf, 2017; Wang, 2011), ya que es fácil de entender, calcular y se puede aplicar a más de dos codificadores. La fórmula es: $P A O=2 A /(N 1+N 2)$ donde $P A 0$ representa el porcentaje de acuerdo entre dos codificadores, $A$ es el número de decisiones de consenso de dos codificadores, y N1 y N2 son los números de decisiones que los codificadores han tomado respectivamente (Wang, 2011). Sin embargo, dado que queremos conocer el grado de acuerdo de los tres investigadores, tuvimos que modificar esta fórmula quedando $P A O=3 \mathrm{~A} /$ $(N 1+N 2+N 3)$ donde $P A 0$ representa el porcentaje de acuerdo entre tres codificadores, $A$ es el número de decisiones de consenso de los tres codificadores, y N1, N2 y N3 son los números de decisiones que los codificadores han tomado respectivamente.

El índice Holsti de nuestro sistema de categorías fue de 0,92 . Sin embargo, siguiendo las recomendaciones de Neuendorf (2017), decidimos realizar el Holsti por categorías (Tabla 3) para tener información específica de cada una y evitar que el promedio de acuerdos ocultara la baja fiabilidad de alguna de ellas. Como se puede apreciar,
Tabla 3. Holsti por categorías de los tres investigadores

\begin{tabular}{lcccccccc}
\hline \multicolumn{1}{c}{ Categoría } & LO & LP & LI & CV & DX & DY & DG & CLDC \\
\hline Porcentaje & 90 & 88 & 92 & 94 & 96 & 100 & 90 & 96 \\
Categoría & PR & NA & ANR & RV & S & G & PM & Global \\
Porcentaje & 98 & 92 & 92 & 88 & 86 & 90 & 92 & 92 \\
\hline
\end{tabular}

Nota: Fuente propia de la investigación. 
cada categoría tiene un porcentaje alto de acuerdo: símbolos matemáticos y notaciones (S) es la categoría con el porcentaje más bajo; descoordinación de los procesos en el rango de la función (DY) es la categoría con el porcentaje más alto de acuerdo. Se aprecia cómo de las 15 categorías 12 tienen un acuerdo entre los tres investigadores del 0,90 o más. Se debe indicar que el índice Holsti de la primera versión aplicada a los estudiantes de Biología arrojó un Holsti global de los tres investigadores de 0,80 .

\section{Análisis y resultados}

Como se comentó en la metodología, se pidió a los estudiantes que explicaran con sus palabras el significado de la definición de límite, con el objetivo de determinar, en aspectos generales, la forma en que comprendían dicho concepto y los aspectos o características que resaltaban de este mismo. Las respuestas de los sujetos de investigación se pudieron ubicar en al menos una de las quince categorías descritas. Mostramos, a continuación, los resultados de dicha codificación.

\section{Categorías: Límite como objeto (LO) y límite como proceso (LP)}

Los sujetos de investigación evidenciaron únicamente la categoría límite como objeto en un $8 \%$ de las respuestas. Entre las respuestas más usuales para esta categoría, sobresalen las siguientes palabras para referirse al límite: es $L$, imagen, el fin, lugar, número, valor, valores, punto, cero, cuatro, huecos, existe, hasta donde llega la función. La presencia de esta categoría se evidencia en la respuesta de EBM0606: El límite de una función es el lugar por donde esta no pasa (en la gráfica). Para que este exista es necesario que los límites laterales se acerquen a un número en común.

Los sujetos de investigación evidenciaron únicamente la categoría límite como proceso en un $49 \%$ de las respuestas. Para esta categoría sobresalen las siguientes palabras para referirse al límite: aproximarse, aproxima, tendencia, tiende, tienden, acercando, acerca, acercar, al acercarse, acercamiento, acerquen, acercándose, cercanos, aproxima, aproximan, aproximaciones, es una proximidad, estudio de valores muy cercanos a un número, sustituyo valores en una variable específica, revelan el comportamiento cerca de un punto dado, analizar lo que hay alrededor de un punto, lo que está pasando alrededor de un punto $x$, se busca llegar, buscar números cercanos, análisis de un punto, aproxima, cada vez más cercano, lo que sucede cerca de un punto, se estudia el alrededor de los puntos. La presencia de esta categoría se aprecia en la respuesta de EQH0110: Es cuando tomamos un número real a del eje $x$ y analizamos cuáles son las imágenes de los números enteros cercanos a a, sin contar a este.

Finalmente, los sujetos de investigación evidenciaron simultáneamente ambas categorías en un $37 \%$ de las respuestas. Podemos notar la presencia de ambas categorías simultáneamente en la respuesta de EBH0701: Un valor aproximado al punto, donde hay una tendencia. Luego se desprende que, el límite como objeto fue seleccionado en un $45 \%$ de las respuestas y el límite como proceso fue seleccionado en un $88 \%$.

\section{Categoría: Vinculación entre límite e imagen (LI)}

La vinculación entre el límite e imagen fue evidenciada en un $14 \%$ de las respuestas por los sujetos de investigación. Entre las respuestas más usuales para esta categoría 
destacan las frases: las imágenes de $a$ en $y$ tienden a $L$, esta imagen es el límite, el valor que pertenece a una función, cuando sustituyo valores en una variable específica, es el valor que toma la $y$, el límite de $f(x)$ es 4 , es un punto al cual se acerca ubicado en $f(x)$.

La presencia de esta categoría se aprecia en la respuesta de EQH0221: El límite de una función es el valor que toma la y al acercarse la x por la izquierda y la derecha en la recta.

\section{Categorías: Coordinación entre las} variables $x$ e $y(C V)$, descoordinación de los procesos en el dominio de la función (DX), descoordinación de los procesos en el rango de la función (DY), descoordinación general de los procesos (DG)

La coordinación entre las variables $x$ e $y$ fue evidenciada en un $22 \%$ de las respuestas por los sujetos de investigación. La presencia de esta categoría se evidencia en la respuesta de EQM0807: El límite de una función es cuando tanto por la derecha como por la izquierda de x se acerca a un determinado punto en y. Es una proximidad.

La descoordinación de los procesos en el dominio de la función fue evidenciada en un $6 \%$ de las respuestas por los sujetos de investigación. Para esta categoría destacan las frases: tiende al acercarse a un valor en el eje $x, x$ se acerca a un número, $x$ tiende a cualquier número. La presencia de esta categoría se aprecia en la respuesta de EQM0213: Cuando en una función x se acerca a un número y no da ese número, el límite de esa función es ese mismo número.

La descoordinación de los procesos en el rango de la función se encontró únicamente en la respuesta de EBH0619: El límite es una tendencia hacia un número sin que este esté incluido. Es el acercamiento de la imagen a un número, quien hace referencia al acercamiento de la imagen a un número.

Finalmente, la descoordinación general de los procesos fue evidenciada en un $57 \%$ de las respuestas por los sujetos de investigación. Para esta categoría destacan las frases: acercamiento de los valores de una función hacia el límite, se acerquen a un número en común, un valor aproximado al punto donde hay una tendencia, estudio de valores muy cercanos a un número, no se encuentra el número como tal sino que es deducible por una tendencia, revelan el comportamiento de una función cerca de un punto, son aproximaciones, analizar lo que hay alrededor de un punto, tendencia de una variable a tomar un valor definido, lo que sucede cerca de un punto pero no exactamente en él [sic], solo se permite acercarse a cierto valor, aquel número cuyo valor se aproxima, el límite de $x$ es un punto al cual se acerca, se estudia alrededor de los puntos, es cuando una función en su gráfica se acerca a un punto, es el punto en una gráfica al que tiende la función, saber a qué número se aproxima, se aproxima a un mismo número, la aproximación de un número. La presencia de esta categoría se evidencia en la respuesta de EBH0521: Se refiere a un acercamiento de los valores de una función hacia el límite, el cual varía de longitud, posición y tendencia a medida que se va acercando al punto final de la función.

\section{Categorías: Condiciones de laterali- dad y doble convergencia (CLDC) y términos de posición relativa $(\mathrm{PR})$}

Las condiciones de lateralidad y doble convergencia fueron evidenciadas en un 24 $\%$ de las respuestas por los sujetos de investigación. Entre las respuestas más usuales para esta categoría destacan las frases: si al aproximarse a $a$ por derecha y por izquierda 
se obtiene el resultado; es el límite si es la misma cuando se acerca tanto por derecha como por izquierda de la preimagen; es necesario que los límites laterales se acerquen a un número en común; tiende al acercarse a un valor en el eje $x$ tanto acercándose por ambos lados, toma un valor en el eje $x$; es el valor que toma la $y$ al acercarse la $x$ por la izquierda y la derecha en la recta; en un límite se buscan aquellos números aproximados a $y$ sea por la izquierda o por la derecha; un límite existe cuando los valores concuerdan por la izq y derecha; es el punto en una gráfica al que tiende la función tanto por la izquierda como por la derecha; saber a qué número se aproxima la función por la derecha y la izquierda; es cuando tanto por la derecha como por la izquierda de $x$ se acerca a un determinado punto en $y$; es cuando tanto por la derecha como por la izquierda de $x$ las $y$ se acercan o aproximan a un determinado punto; ya sea por la izquierda o por la derecha, el límite se aproxima a un mismo número. La presencia de esta categoría se evidencia en la respuesta de EQH0813: El límite de una función es hasta donde llega la función, ya sea por la izquierda o por la derecha, el límite se aproxima a un mismo número.

Los términos de posición relativa fueros evidenciados en un $51 \%$ de las respuestas por los sujetos de investigación. Para esta categoría sobresalen las siguientes frases: izquierda y derecha de $a$, derecha como por izquierda de la preimagen, valor más próximo al punto, valor aproximado al punto, valores muy cercanos a un número, cerca de un punto, por ambos lados se acerca a un valor en el eje $x$, alrededor de un punto, alrededor de un punto $x$, números enteros cercanos a $a$, por la izquierda y la derecha, por la izquierda o por la derecha, por ambos lados (izquierda, negativo y derecha, positivo), cerca de un valor $x$, cercano a 0 , alrededor de los puntos, valores cercanos a un punto, izquierda como por la derecha, es una proximidad. Como se mencionó en la descripción de estas categorías, en las respuestas donde hay evidencia de CLDC hay evidencia de PR, pero no viceversa, un ejemplo de este hecho es respuesta de EBM0927 (1) Los límites revelan el comportamiento de una función cerca de un punto dado.

\section{Categorías: Aspectos estructurales de no alcanzabilidad (NA) y aspec- tos estructurales de alcanzabilidad y no rebasabilidad (ANR)}

Los aspectos estructurales de no alcanzabilidad fueron evidenciados en $12 \%$ de las respuestas por los sujetos de investigación. Entre las respuestas más usuales para esta categoría sobresalen las frases: es el lugar por donde esta no pasa (en la gráfica), sin que este esté incluido, pero siguiendo la regla de que $y$ no va a ser el resultado, sin nunca tocar dicho valor, pero nunca lo llega a pasar o tocar, se acerca a un punto pero nunca lo toca. La presencia de esta categoría se evidencia en la respuesta de EQH0205: Significa que cuando $x$ se aproxima a un valor simultáneamente se acerca a un valor de $f(x)$ sin nunca tocar dicho valor.

Los aspectos estructurales de alcanzabilidad $\mathrm{y}$ no rebasabilidad fueron evidenciados en un $8 \%$ de las respuestas por los sujetos de investigación. Para esta categoría destacan las frases: punto final, valor máximo, solo se le permite acercarse a cierto valor no pasarse de él, el límite de una función es hasta dónde [sic] llega la función. La presencia de esta categoría se evidencia en la respuesta de EBH0701: Es el valor máximo o más próximo al punto que pertenece a una función. 


\section{Categoría: Referencia a las variables $x$ e y (RV)}

La referencia a las variables $x$ e $y$ fue evidenciada en un $31 \%$ de las respuestas por los sujetos de investigación. Entre las respuestas más frecuentes para esta categoría destacan las siguientes expresiones: preimagen, imagen, imágenes, valor en el eje $x$, punto $x$, punto en $y$, valor que toma la $y$, valores de $y$, números en $x$, número $y$, $x, y, f(x)$. La presencia de esta categoría se evidencia en la respuesta de EBH0619: El límite es una tendencia hacia un número sin que este esté incluido. Es el acercamiento de la imagen a un número.

\section{Categorías: Símbolos matemáticos y notaciones (S) y referencia a un sistema de representación gráfico o geométrico $(\mathbf{G})$}

Los símbolos matemáticos y notaciones fueron evidenciados en un $35 \%$ de las respuestas por los sujetos de investigación. Entre las respuestas más usuales para esta categoría sobresalen los siguientes símbolos: $f, a, L, y, x, 0 / 0, y^{-, y^{+}}, \lim _{\mathrm{x} \rightarrow \mathrm{a}} f(x)=y$, $f(x), 2,3,4,1,1 / 2,1 / 3 \ldots, 1,1 / 4,1 / 9 \ldots . \mathrm{La}$ presencia de esta categoría se evidencia en la respuesta de EQM0223: Significa que por ejemplo si $x$ tiende a 2 y $f(x)$ a 4 , el límite de $f(x)$ cuando $x$ tiende a 2 es igual a 4.

La referencia a un sistema de representación gráfico o geométrico, es decir, fue evidenciada en un $22 \%$ de las respuestas por los sujetos de investigación. Para esta categoría, entre las respuestas más habituales que emplearon los sujetos destacan las palabras: punto final, en la gráfica, el punto en una recta, gráficamente, una recta tiene un espacio o punto sin nada como hueco en medio, un punto en una gráfica, una recta o plano, huecos que hay en un gráfico, gráfica se acerca a un punto, gráfica, forma. La presencia de esta categoría se evidencia en la respuesta de EQM0518: Es cuando una función en su gráfica se acerca a un punto, pero nunca lo toca. Son valores cercanos a un punto en especifico.

\section{Categoría: Propiedades matemáti- cas (PM)}

Las propiedades matemáticas fueron evidenciadas en un $8 \%$ de las respuestas por los sujetos de investigación. Entre las respuestas más frecuentes para esta categoría destacan las siguientes frases: función real de variable real, hay límites infinitos, de manera que se evite el resultado $0 / 0$, sucesiones, límite de sucesiones. La presencia de esta categoría se evidencia en la respuesta de EBM1322: Es un número al que tiende una función. Hay límites infinitos.

Presentamos en la tabla 4 un resumen del porcentaje de evidencia de cada categoría, basado en las 49 respuestas de los estudiantes.

Como se puede apreciar en la Tabla 4 , el $65 \%$ de las respuestas de los estudiantes evidencian al límite como proceso, pero presentan descoordinación en algún sentido: en el dominio de la función, en el rango de la función o, en general, en los procesos.

Tabla 4. Porcentaje de evidencia de cada categoría, basado en las 49 respuestas de los estudiantes

\begin{tabular}{lcccccccc}
\hline \multicolumn{1}{c}{ Categoría } & LO & LP & LI & CV & DX & DY & DG & CLDC \\
\hline Porcentaje & 45 & 88 & 14 & 22 & 6 & 2 & 57 & 24 \\
Categoría & PR & NA & ANR & RV & S & G & PM & \\
Porcentaje & 51 & 12 & 8 & 31 & 35 & 22 & 8 & \\
\hline
\end{tabular}

Nota: Fuente propia de la investigación. 
En el siguiente apartado, presentamos una síntesis y concluimos sobre los principales resultados obtenidos en el estudio. Esto corresponde a las fases 6 y 7 del análisis de contenido.

\section{Conclusiones}

En este trabajo nos planteamos dos objetivos. El primero de ellos consistió en realizar el diseño y validación de un sistema de categorías para el análisis de definiciones de límite de una función en un punto, basadas en el trabajo de Fernández-Plaza et al. (2013). En cuanto a este objetivo destacamos que hemos realizado un método sistemático como lo es el análisis de contenido que hemos organizado en fases (Figura 2). Puntualmente, la reiteración de las fases 4 y 5 nos permitió tener un sistema de categorías fiable, debido a que tenemos un índice de acuerdo entre investigadores de un $92 \%$ $\mathrm{y}$, específicamente, de las 15 categorías, 12 obtienen más de un $90 \%$ de acuerdo entre los tres investigadores y las otras 3 entre un $86 \%$ y un $88 \%$. Este índice de acuerdo nos permite concluir que los resultados obtenidos son fiables. Es decir, el detalle con el que son descritas las categorías y el proceso de análisis proporciona información suficiente para replicarlo. Además, se describe la forma de proceder a la hora de diseñar y validar el proceso, mostrando un procedimiento válido para crear categorías de análisis de definiciones de otros conceptos o tareas diferentes. Con nuestro estudio, complementamos el trabajo de Fernández-Plaza et al. (2013), al aportar más categorías, que ponemos a prueba en otro contexto y con otros sujetos de investigación.

Consideramos que el sistema de categorías que hemos creado nos permitirá analizar más unidades de información de una manera sistemática y simple. En general, creemos que el análisis de contenido que hemos realizado puede permitir a futuros investigadores crear un sistema de categorías similar o usar este para investigaciones similares en otros contextos.

El segundo objetivo de nuestro trabajo consistió en utilizar el sistema de categorías para analizar las definiciones del concepto de límite proporcionadas por 38 estudiantes universitarios de Biología e Ingeniería en Química Industrial de la Universidad Nacional en Costa Rica.

Resaltamos la concepción dual objeto/proceso que atribuyeron los sujetos al límite, pues aproximadamente en la mitad de las respuestas lo señalaron como objeto y en más de tres cuartas partes de las respuestas lo señalaron como proceso en la definición, esta concepción dual que atribuyen los sujetos al límite también la evidencian en sus estudios Fernández-Plaza et al. (2013), Tall (1980) y Sfard (1991).

Asimismo, aproximadamente en la mitad de las respuestas los sujetos evidencian la categoría descoordinación general de los procesos, en una cuarta parte muestran la categoría coordinación entre las variables $x$ e $y$, y en una décima parte la categoría descoordinación de los procesos en el dominio de la función, y la categoría descoordinación de los procesos en el rango de la función.

Luego, en más de la mitad de las respuestas los sujetos evidencian la categoría términos de posición relativa, en aproximadamente una cuarta parte de las respuestas, los sujetos evidencian las categorías condiciones de lateralidad y doble convergencia, referencia a las variables $x$ e $y$, símbolos matemáticos y notaciones, $\mathrm{y}$ referencia a un sistema de representación gráfico o geométrico. 
Finalmente, cerca de una décima parte de las respuestas de los sujetos resaltan las categorías vinculación entre límite e imagen, propiedades matemáticas, aspectos estructurales de no alcanzabilidad, y aspectos estructurales de alcanzabilidad y no rebasabilidad.

Una de las concepciones que manifestaron los estudiantes, aunque en menor medida, fue la del límite como noción inalcanzable, en lo cual se coincide con Williams (1991) y con Cornu (2002). Asimismo, un número pequeño de estudiantes evidenciaron una concepción de alcanzabilidad y no rebasabilidad del límite, así se concuerda con Fernández-Plaza et al. (2013).

\section{Reconocimientos}

Este trabajo fue desarrollado dentro del marco del Proyecto I+D+I PCG2018095765-B-100 del Ministerio de Ciencia y Tecnología de España y del Grupo FQM193 del III Plan Andaluz de Investigación (PAIDI).

\section{Consentimiento informado}

Los autores declaramos que los participantes de este estudio fueron informados sobre el tratamiento de la información.

\section{Conflicto de intereses}

Los autores declaran no tener conflicto de interés.

\section{Declaración de la contribución de los autores}

El porcentaje total de contribución para la conceptualización, preparación y corrección de este artículo fue el siguiente: Y.G.F. 50 \%, A.M.M. 25 \% y J.R.H. $25 \%$.

\section{Declaración de disponibilidad de los datos}

Los datos que respaldan los resultados de este estudio serán puestos a disposición por el autor correspondiente [Y.G.F.], previa solicitud razonable.

\section{Referencias}

Artigue, M. (1995). La enseñanza de los principios del cálculo: Problemas epistemológicos, cognitivos y didácticos. En M. Artigue, R. Douady, L. Moreno y P. Gómez (Eds.), Ingeniería didáctica en educación matemática (pp. 97-140). Grupo Editorial Iberoamérica. http://funes.uniandes. edu.co/676/1/Artigueetal195.pdf\#page $=105$

Azcárate, C. y Camacho, M. (2003). Sobre la investigación en didáctica del análisis. Boletín de la Asociación Matemática Venezolana, 10(2), 135- 149.

Bell, A. W., Costello, J. \& Küchemann, D. E. (1983). A review of research in mathematical education: research on learning and teaching. The NFER-NELSON Publishing Company.

Blázquez, S. (1999). Sobre la noción del límite en las matemáticas aplicadas a las ciencias sociales. En T. Ortega (Ed.), Investigación en educación matemática III (pp. 167-184). SEIEM.

Blázquez, S. (2000). Noción de límite en matemáticas aplicadas a las ciencias sociales [Tesis doctoral no publicada]. Universidad de Valladolid.

Blázquez, S. y Ortega, T. (1998). Rupturas en la comprensión del concepto de límite en alumnos de bachillerato. Aula, 10, 119-135. https:/gredos.usal.es/bitstream/handle/10366/69322/ Rupturas_en_la_comprension_del_concepto .pdf? sequence $=1$ \&isAllowed $=\mathrm{y}$

Blázquez, S. y Ortega, T. (2002). Nueva definición de límite funcional. UNO, 30, 67-82.

Byerley, C. y Thompson, P. (2017). Secondary mathematics teachers' meanings for measure, slope, and rate of change. Journal of Mathematical Behavior, 48, 168-193. https://doi. org/10.1016/j.jmathb.2017.09.003 
Castro, E. y Castro, E. (1997). Representaciones y modelización. En L. Rico (Ed.), La educación matemática en la enseñanza secundaria (pp. 95-124). Horsori.

Cohen, L., Manion, L. y Morrison, K. (2007). Research Methods in Education (sixth edition). Routledge. https://doi.org/10.4324/9780203029053

Cornu, B. (2002). Limits. In D. Tall (Ed.), Advanced Mathematical Thinking (pp. 153-166). https://doi.org/10.1007/0-306-47203-1_10

Fernández-Plaza, J. (2016). Análisis del contenido. En L. Rico y A. Moreno (Eds.), Elementos de didáctica de la matemática para el profesor de secundaria (pp. 103-118). Ediciones Pirámide.

Fernández-Plaza, J. A., Ruiz-Hidalgo, J. F., Rico, L. y Castro, E. (2013). Definiciones personales y aspectos estructurales del concepto de límite finito de una función en un punto. PNA, 7(3), 117-130. https://digibug.ugr.es/bitstream/ handle/10481/23475/PNA7\%283\%29-3. pdf? sequence $=1 \&$ isAllowed $=\mathrm{y}$

Hernández, R., Fernández, C. y Baptista, P. (2014). Metodología de la investigación (Sexta edición). McGraw Hill.

Hiebert, J. y Lefevre, P. (1986). Conceptual and Procedural Knowledge in Mathematics: An Introductory Analysis. En J. Hiebert (Ed.), Conceptual and Procedural Knowledge in Mathematics: The case of Mathematics (pp. 1-28). Lawrence Erlbaum Associates.

Juter, K. (2007a). Students 'Concept Development of Limits. Proceedings of the Fifth Congress of the European Society for Research in Mathematics Education (CERME). Working group 14, 2320-2329.

Juter, K. (2007b). Students'Conceptions of Limits: High Achievers versus Low Achievers. The Montana Mathematics Enthusiast (TMME), 4(1), 53-65. https://www.diva-portal.org/ smash/get/diva2:207620/FULLTEXT01.pdf

Kaput, J. (1987). Representations Systems and Mathematics. En C. Janvier (Ed.), Problems of representation in the teaching and learning of mathematics (pp. 19-26). Lawrence Erlbaum Associated.

Kidron, I. (2014). Calculus teaching and learning. Encyclopedia of mathematics education. (pp.69-75). Springer. https://doi.org/10.1007/978-94-007-4978-8

Kidron, I., \& Tall, D. (2014). The roles of visualization and symbolism in the potential and actual infinity of the limit process. Educational Studies in Mathematics, 88, 183-199. https://doi. org/10.1007/s10649-014-9567-x
Kilpatrick, J., Hoyles, C. y Skovsmose, O. (Eds.) (2005). Meanings of meaning of mathematics. En Meaning in Mathematics Education (pp. 9-16). Springer. https://doi. org/10.1007/0-387-24040-3 2

Krippendorff, K. (2004). Content analysis: An introduction to its methodology (Second Edition). Sage publications.

Monaghan, J. (1991). Problems with the Language of Limits. For the Learning of Mathematics, 11(3), 20-24. https://flm-journal.org/Articles/ 3905D8E2472320B3602153840B1E86.pdf

Neuendorf, K. (2017). The Content Analysis Guidebook (Second Edition). Sage publications. https://doi.org/10.4135/9781071802878

Rico, L. (1997). La educación matemática en la enseñanza secundaria. En L. Rico, E. Castro, M. Coriat, A. Marín, L. Puig, M. Sierra, M. M. Socas (Eds.), Consideraciones sobre el currículo de matemáticas para educación secundaria (pp. 15-38). ice - Horsori.

Rico, L. (2012). Aproximación a la investigación en didáctica de la matemática. Avances de Investigación en Educación Matemática, 1, 39-63. https://doi.org/10.35763/aiem.v1i1.4

Rico, L. (2013). El método del análisis didáctico. Revista Iberoamericana de Educación Matemática, 33, 11-27. http://funes.uniandes.edu. co/15988/1/Rico2013El.pdf

Rico, L. (2016a). Matemática y análisis didáctico. En L. Rico y A. Moreno (Eds.), Elementos de didáctica de la matemática para el profesor de secundaria (pp. 85-100). Ediciones Pirámide.

Rico, L. (2016b). Significados de los contenidos matemáticos. En L. Rico y A. Moreno (Eds.), Elementos de didáctica de la matemática para el profesor de secundaria (pp. 153174). Ediciones Pirámide.

Rico, L. y Fernández-Cano, A. (2013). Análisis didáctico y metodología de investigación. En L. Rico., J. Lupiañez. y M. Molina (Eds.), Análisis didáctico en educación matemática: Metodología de investigación, formación de profesores e innovación curricular (pp.1-22). Comares.

Rico, L. \& Ruiz-Hidalgo, J. F. (2018). Ideas to Work for the Curriculum Change in School Mathematics. En Y. Shimizu y R. Vital (Eds.), ICMI Study 24 Conference proceedings. School Mathematics Curriculum Reforms: Challenges, Changes and Opportunities (pp. 301-308). ICMI.

Romero, I. (1997). La introducción del número real en educación secundaria: Una experiencia de investigación-acción. Comares. 
Ruiz-Hidalgo, J. (2016). Sentido y modos de uso de un concepto. En L. Rico y A. Moreno (Eds.), Elementos de didáctica de la matemática para el profesor de secundaria (pp. 139151). Ediciones Pirámide.

Sierpinska, A. (1987). Humanities students and epistemological obstacles related to limits. $E d u$ cational Studies in Mathematics, 18(4), 371397. https://doi.org/10.1007/BF00240986

Sfard, A. (1991). On the dual nature of mathematical conceptions: reflections on processes and objects as different sides of the same coin. $E d u$ cational Studies of Mathematics, 22, 1-36. https://doi.org/10.1007/BF00302715

Sierra, M., González, M. y López, C. (2000). Concepciones de los alumnos de bachillerato y curso de orientación universitaria sobre límite funcional y continuidad. Revista Latinoamericana de Investigación en Matemática Educativa, 3(1), 71-85.

Sim, J., \& Wright, C. (2005). The Kappa Statistic in Reliability Studies: Use, Interpretation, and Sample Size Requirements. Physical Therapy, 85(3), 257-268. https://doi.org/10.1093/ptj/85.3.257

Steinbring, H. (1997). Epistemological investigation of classroom interaction in elementary mathematics teaching. Educational Studies in Mathematics, 32(1), 49-92. https://doi. org/10.1023/A:1002919830949

Steinbring, H. (2006). What makes a sign a mathematical sign? - An epistemological perspective on mathematical interaction. Educational Studies in Mathematics, 61, 133-162. https:// doi.org/10.1007/s10649-006-5892-z

Swinyard, C. (2011). Reinventing the formal definition of limit: The case of Amy and Mike, The Journal of Mathematical Behavior, 7(4), 765 790. https://www.sciencedirect.com/science/ article/pii/S0732312311000022

Tall D. O. (1980). Mathematical intuition, with special reference to limiting processes. In $\mathrm{R}$. Karplus (Ed.), Proceedings of the Fourth International Conference for the Psychology of Mathematics Education (pp. 170-176). PME.

Tall, D \& Vinner, S. (1981). Concept image and concept definition in mathematics, with special reference to limits and continuity, Educational Studies in Mathematics, 12, 151- 169. https://doi.org/10.1007/BF00305619

Tall, D., \& Katz, M. (2014). A cognitive analysis of Cauchy's conceptions of function, continuity, limit, and infinitesimal, with implications for teaching the calculus. Educational Studies in Mathematics, 86(1), 97-124. https://doi. org/10.1007/s10649-014-9531-9

Thompson, P. (2013). In the absence of meaning.... En K. Leatham (Ed.), Vital directions for research in mathematics education (pp. 57-93). Springer. https://doi. org/10.1007/978-1-4614-6977-3 4

Thompson, P. (2016). Researching mathematical meanings for teaching. En L. English y D. Kirshner (Eds.), Handbook of International Research in Mathematics Education (pp. 435-461). Taylor and Francis.

Thompson, P. y Milner, F. (2019). Teachers' Meanings for Function and Function Notation in South Korea and the United States. En H. G. Weigand, W. McCallum, M. Menghini, M. Neubrand y G. Schubring (Eds.), The Legacy of Felix Klein (pp. 55-66). Springer. https:// doi.org/10.1007/978-3-319-99386-7_4

Vergnaud, G. (2009). The Theory of Conceptual Fields. Human Development, 52, 83-94. https://doi.org/10.1159/000202727

Vergnaud, G. (2013). Conceptual development and learning. Revista Curriculum, 26, 39-59. https://qurriculum.webs.ull.es/0_materiales/articulos/Qurriculum\%2026/Qurriculum\%2026-2013(3).pdf

Vrancken, S., Gregorini, M. I., Engler, A., Muller, D., \& Hecklein, M. (2006). Dificultades relacionadas con la enseñanza y el aprendizaje del concepto de límite. Revista PREMISA, 8(29), 9-19.

Wang, W. (2011). A Content Analysis of Reliability in Advertising Content Analysis Studies [Master's Thesis]. East Tennessee State University.

Williams, S. (1991). Models of limit held by college calculus students. Journal for research in $\mathrm{Ma}$ thematics Education, 22(3), 219-236. https:// doi.org/10.5951/jresematheduc.22.3.0219

\section{(c) $(i)$}

Análisis de las definiciones de límite que brindan estudiantes universitarios (Yosenith González-Flores • Ana Belén Montoro-Medina • Juan Francisco Ruiz-Hidalgo) Uniciencia is protected by Attribution-NonCommercial-NoDerivs 3.0 Unported (CC BY-NC-ND 3.0) 